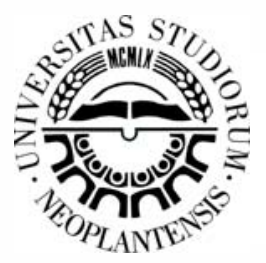

\title{
Development of a self-optimizing baking system
}

\author{
Lucijano Berus, * Simon Klancnik, Timi Karner, Mirko Ficko \\ University of Maribor, Faculty of Mechanical Engineering, Maribor, Slovenia
}

\begin{abstract}
This study focuses on designing a protocol for optimization of temperature variances inside heating device. Protocol consists of two major parts. First one is the protocol for monitoring temperature distributions and managing heat sources inside an oven. Second part is the optimization scheme, responsible for minimizing the distribution of temperature. Thermocouple J-type sensors are distributed on horizontal plane inside an oven. Digitally operated relay module switch enables heaters manipulation by pulse-width modulation (PWM) signal. Monitoring and operating of the system components is enabled by to data acquisition (DAQ) device and PC, performing the optimization with Particle swarm optimization algorithm. PSO objective function represented as maximum temperature difference recorded by sensors inside heating device is optimized. Included safety protocol serves as an off-switch if temperature inside the oven reaches critical level.
\end{abstract}

Key words: oven; temperature monitoring; particle swarm optimization algorithm; latin hypercube sampling;

\section{INTRODUCTION}

In cooking, the conventional oven is a kitchen device, used by many households for heating and roasting. Foods normally cooked in this manner include meat, bread, cake and other desserts. Outside the culinary world, heating devices are used also for melting (furnace for steel manufacture) and drying (kilns for ceramics, wood and cement manufacturing). For controlling the temperature conditions inside these kind of heating devices companies have adopted different techniques. Some have resolved oven issues with supervisory control and data acquisition systems, both on premise and even remotely. Other manufactures have adopted other kind of computer software, such as line cameras to monitor and provide remote assistance or even fix problems around the world $[1,2]$.

Food industry in recent years demands products with better control to provide good and acceptable dishes. The quality of food is influenced by heating device properties, such as distribution of heaters, fan properties, outside conditions, materials used and many more. Backing operation efficiency is determined by the oven properties and operating conditions [3]. Baking is a simultaneous heat and mass transfer phenomena (heat is transferred with conduction, convection and radiation) [4], it's a non- reversible process, thus any unsatisfactory product has to be discharged. Baking is a very complex process, during which products are exposed to numerous physical, chemical and biochemical changes, such as water and fat phase transition, denaturation of protein, crust formation, water evaporation, volume expansion, browning reaction, generation of porous structure etc. Temperature and water content are responsible for all physiochemical processes associated with baking process and variations in the product can potentially be minimalized by optimizing the processing conditions [5]. Heating process could produce a non-uniform temperature distribution within the food, possibly resulting in under-cooked and over-cooked regions [6]. Many factors affect uniformity of baking, including: thermal properties (specific heat capacity and thermal conductivity) and physical properties (size, shape, density) of foods [7]. There are few parameters that has to be monitored inside a heating device; heat flux, humidity, air velocity and temperature.

This study concentrates on designing a self-learning protocol, that operates the heaters, depicts temperature field and finds an optimal setting for homogenous temperature distribution inside an oven chamber. Research aim is to construct user friendly procedure to control (by manipulating the heaters) and monitor the optimization of air temperature inside the oven. System of this sort enables

* Corresponding author's.e-mail: lucijano.berus@um.si 
the optimization of temperature field variations and is translatable to other kind of devices, that perform heating or cooling processes.

\section{MATERIALS AND METHODS}

\subsection{Sensors}

Sensor is a device whose purpose is the detection of events or changes in the environment. Information about environment is then sent and processed by computer or other type of electronics. Sensors, also called transducers, converts inputs representing the physical phenomena in environment into measurable signal output, that is readable to a human. Temperature sensors monitor free stream air temperature associated with convection. Mathematical models and computer software are used to create temperature graphs. By measuring the temperature one can analyse the precise baking process and use that information to control product quality. Thermocouple temperature sensors work by producing a small electrical current, that is proportional to temperature difference between two ends, named hot and cold junction.

For this study J-type thermocouple sensors were used. Jtype sensor is made of iron and constantan alloy combination and has a temperature range from 0 to 400

C. Sixteen J-type thermocouple sensors are connected to sixteen analogue input ports of DAQ. Temperature difference between cold and hot junction is calculated with following formula:

$\Delta \boldsymbol{T}=\boldsymbol{T}_{\mathbf{0}}+\frac{\left(v-v_{0}\right)\left(p_{1}+\left(v-v_{0}\right)\left(p_{2}+\left(v-v_{0}\right)\left(p_{3}+p_{4}\left(v-v_{0}\right)\right)\right)\right)}{1+\left(v-v_{0}\right)\left(q_{1}+\left(v-v_{0}\right)\left(q_{2}+q_{3}\left(v-v_{0}\right)\right)\right)}$,

where $\boldsymbol{v}$ is the voltage difference between junctions. $\boldsymbol{T}_{\mathbf{0}}$, $\boldsymbol{p}_{\mathbf{1}}, \boldsymbol{p}_{\mathbf{2}}, \boldsymbol{p}_{\mathbf{3}}, \boldsymbol{p}_{\mathbf{4}}, \boldsymbol{q}_{\mathbf{1}}, \boldsymbol{q}_{\mathbf{2}}, \boldsymbol{q}_{\mathbf{3}}$ and $\boldsymbol{v}_{\mathbf{0}}$ represent specific JType coefficients stated in Table 1 . Real temperature in the oven chamber is calculated with summation of ambient temperature $\boldsymbol{T}_{\text {ambient }}$ and temperature difference $\Delta \boldsymbol{T}$.

Table 1 - J-type thermocouple sensor coefficients.

\begin{tabular}{lr}
\hline & Coefficients \\
\hline $\boldsymbol{T}_{\mathbf{0}}$ & $2.5066947 \mathrm{E}+02$ \\
$\boldsymbol{p}_{\mathbf{1}}$ & $1.8014787 \mathrm{E}+01$ \\
$\boldsymbol{p}_{\mathbf{2}}$ & $-6.5218881 \mathrm{E}-02$ \\
$\boldsymbol{p}_{\mathbf{3}}$ & $-1.2179108 \mathrm{E}-02$ \\
$\boldsymbol{p}_{\mathbf{4}}$ & $2.0061707 \mathrm{E}-04$ \\
$\boldsymbol{q}_{\mathbf{1}}$ & $-3.9494552 \mathrm{E}-03$ \\
$\boldsymbol{q}_{\mathbf{2}}$ & $-7.3728206 \mathrm{E}-04$ \\
$\boldsymbol{q}_{\mathbf{3}}$ & $1.6679731 \mathrm{E}-05$ \\
$\boldsymbol{v}_{\mathbf{0}}$ & $1.3592329 \mathrm{E}+01$ \\
\hline
\end{tabular}

Fig. 1 shows the placement of sixteen sensors inside an oven chamber. For this study single plane arrangement was used. Temperatures were sampled with $1000 \mathrm{~Hz}$ sampling rate.

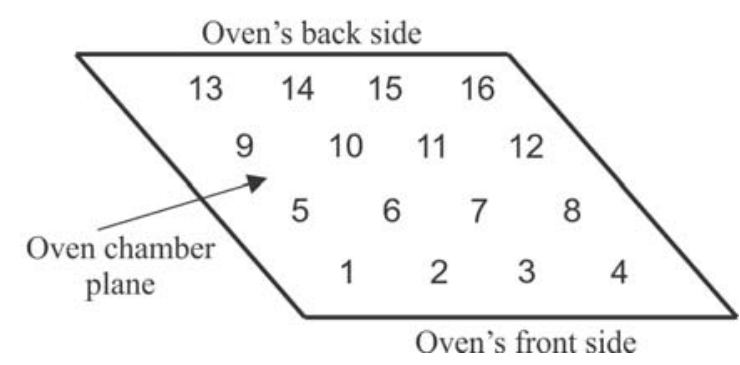

Fig. 1 Sensors numbers and plane arrangement.

\subsection{Relays}

Relay is an electrically operated switch that separates electrical currents. Relays are used where it is necessary to control circuits by a separate low-power signal, or where several circuits must be controlled by one signal. Many relays use an electromagnet to mechanically operate a switch, but there are also other operating principles, such as solid-state relays.

For this study 4 channel 5V relay module was used. Pulsewidth modulation (PWM) signal parameters for operating a relay are period, duty cycle and delay (Fig. 2). On the basis of a proposal of oven manufacturer we decided that we will fixate a period parameter on minimum 45 seconds value, because if the period value was lower, the disturbances on an electric grid would become too significant.

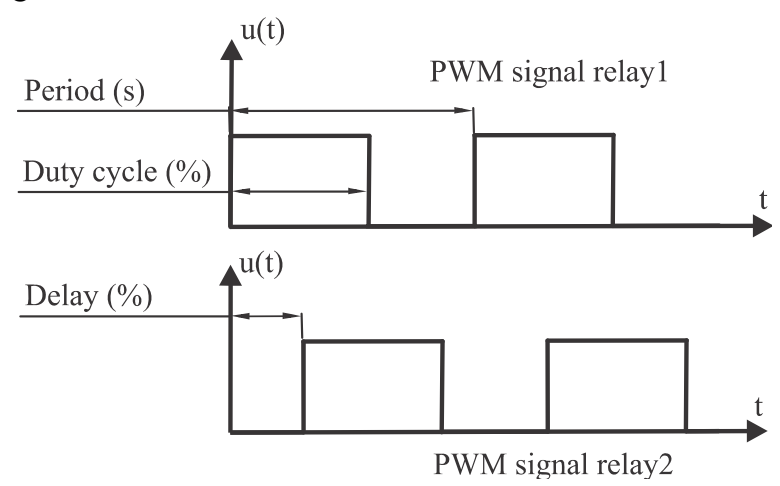

Fig. 2 PWM signal adjustable heater parameters.

Relays are operated with three digital outputs of DAQ device, one relay channel serve as safety switch (if $\boldsymbol{T}_{\text {limit }}$ is reached it cuts down electrical current of other two channels that operate heaters). Fig. 3 shows the circuit connection between heaters and relays.

\subsection{Data acquisition system}

Data acquisition (DAQ) is the process of measuring real world physical phenomena and converting the resulting samples into digital numeric values, that can be manipulated with computer. DAQ systems exploit the processing power, display, connectivity and productivity capabilities of industry standard computers. A DAQ system consists of sensors and actuators, DAQ device, and a computer with programmable software. 

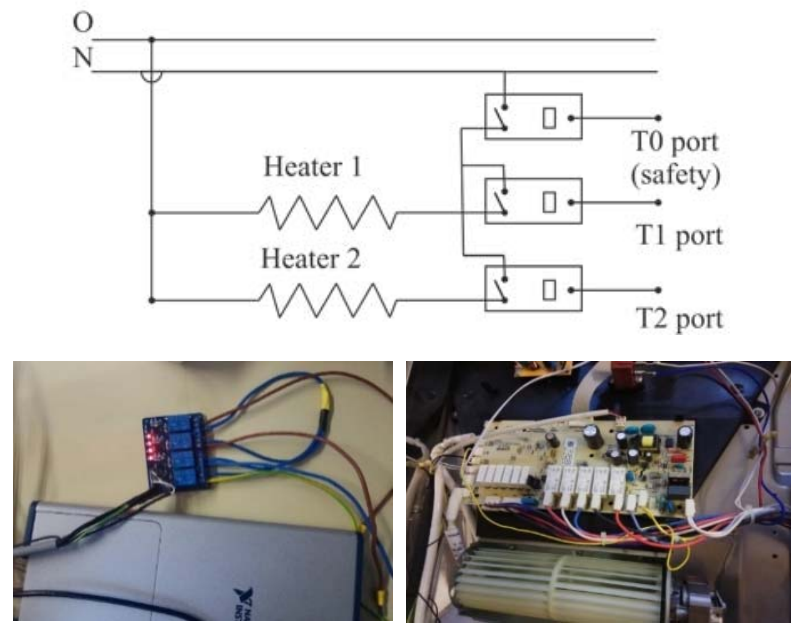

Fig. 3 Circuit connection between heaters and relays.

For this study NI USB-6343 DAQ device was used. DAQ device is connected to PC via USB and manipulated it with software. Sixteen J-type thermocouple sensors were plugged into analogue ports v0 to $\mathbf{v 7}$ and v17 to v24. Relay switches were controlled with 3 digital ports T0 to T2.

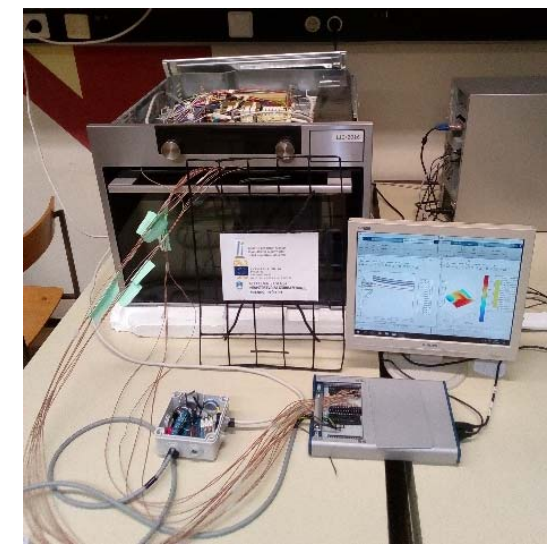

Fig. 4J-type sensors, relay module, ASKO OCS8487S oven, DAQ device and $\mathrm{PC}$

\subsection{Particle swarm optimization}

Optimization is a process of selection the best settings with regard to some criterion. It includes finding the best available values of some objective function, representing the environment. Particle swarm optimization (PSO) is an optimization method, that mimics swarm intelligence, was developed in 1995 by Kennedy and Eberhart [8] and can be adopted to find optimal values in varieties of problems [9]. Algorithm was inspired by a flock of birds, that search for food, which is represented as the optimal value. By default, PSO creates randomly distributed particle positions, that represent certain independent values (in our case PWM signal values). Fig. 5 shows the PSO pseudocode.

Each particle knows it's current position, their best position and global best position. In the first iteration particles positions are generated, with initial velocities set to zero.
Through iteration search particle's velocities $v_{i}^{t}$ are alterd with:

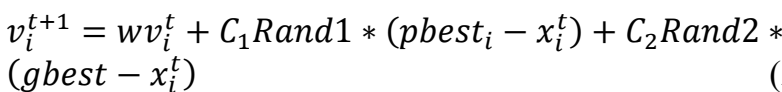

and positions $x_{i}^{t}$ with:

$x_{i}^{t+1}=x_{i}^{t}+v_{i}^{t+1}$,

where $\mathrm{t}$ is the current iteration, $w$ is the inertia coefficient, $C_{1}$ personal coefficient, $C_{2}$ global coefficient, Rand 1 and Rand2 are the random number generators that insure stohasity. The term pbest $_{i}$ allows particle to learn from its own experiences. The gbest term enables certain particle to learn form best experiences of any particle in a swarm.

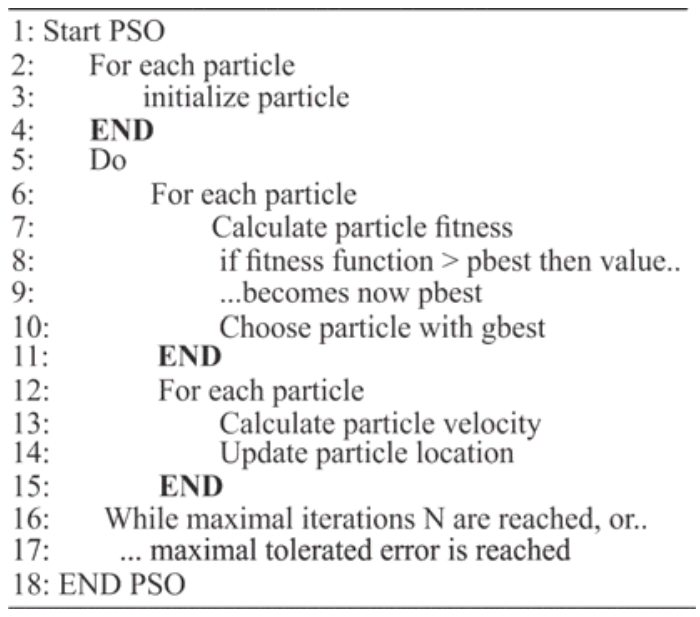

Fig. 5PSO pseudocode [10].

To insure better optimum search, initial particles are generated via. Latin hypercube sampling method (LHS), that was developed by McKay in 1979 [11] and by Eglajs in 1977 [12]. The generation of initial positions with LHS insures better search space division compared to ordinary random sampling with constrains. The convergence rate of PSO is dependent of its hyper parameters. To insure better convergence of $\mathrm{PSO}$, hyper parameters or restriction coefficients $\left(w, C_{1}\right.$ and $\left.C_{2}\right)$ are set according to Kennedy and Clerc recommendation [13].

\section{RESULTS ANDDISCUSSION}

Fig. 6 presents a flowchart of proposed system for monitoring temperature distributions and operating heat sources inside heating device. System enables real time monitoring and altering of the conditions inside an oven chamber.

First the user specifies a desired PWM cycle for heaters (program), working temperature $\mathbf{T}_{\text {working (warm-up), limit }}$ temperature $\mathbf{T}_{\text {limit }}$ and working time. During warm-up procedure heaters are working with full percentage duty cycle (and minimum delay), until the desired $\mathbf{T}_{\text {working }}$ is reached. $\mathbf{T}_{\text {working }}$ is read from desired temperature sensor, in our case we used a sensor no. 4. Then the program (with 
specified PWM signal) starts operating the heaters and simuntaniously gather the temperature field and display it in real-time to the user. Temperature field is shown in a 3D graph and in an ordinary 2D graph that offer a continuous user control of temperature field inside heating device. $\mathbf{T}_{\text {limit }}$ is a part of a safety protocol, that stops the oven if the limiting temperature is surpassed, and informs the user. At the end of working time the temperature field results are exported.

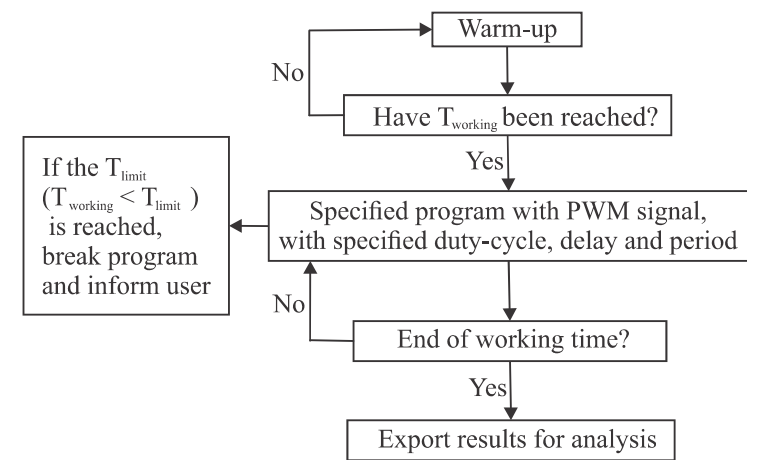

Fig. 6 Workflow of the proposed system for monitoring temperature distributions and managing heat sources.

Fig. 7 shows a 2D temperature graph of temperatures inside oven chamber. Fig. 7 and 8 show a temperature field of PWM signal with properties:

- $\quad$ duty cycle heater $1=\mathbf{2 0} \%$,

- duty cycle heater $2=35 \%$,

- $\quad$ delay heater $1=\mathbf{5 0} \%$,

- delay heater $2=\mathbf{0} \%$,

- $\quad$ period heater $1=\mathbf{4 5 s}$ and

- $\quad$ period heater2 $=\mathbf{4 5 s}$.

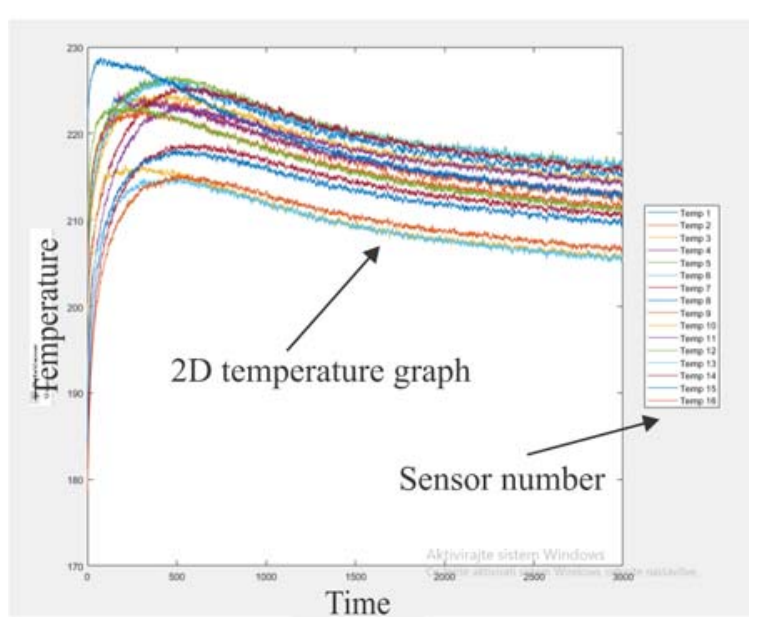

Fig. 72D graph of temperatures inside oven chamber.

Fig. 8 shows a 3D temperature graph field inside oven chamber. With slider the user can visualize a temperature field through time. Sensors are located in the positions as stated in Fig. 1. Sensor no. 1 has position $(\mathbf{1}, \mathbf{1})$, sensor no. 2 position $(2,1)$ and so on up to sensor no. 16 located at position $(\mathbf{4}, \mathbf{4})$. User can also monitor current temperature of all sixteen sensors on the right side of Fig. 8.

The overall workflow of the proposed protocol is shown on
Fig. 9. Depicted protocol consists of system for monitoring temperature distributions and managing heat sources (described in Fig.6) and PSO algorithm, which minimizes the temperature fluctuations inside oven chamber. Fitness of objective function $(F F)$ is defined as:

$F F=|\max T-200|+|\min T-200|$,

where $\max T$ and $\min T$ represent maximum and minimum temperature values, measured among $16 \mathrm{~J}$-type thermocuple sensors. Based on fitness function evaluation of every particle, particle positions are altered. Particle positions are represented as PWM signal.

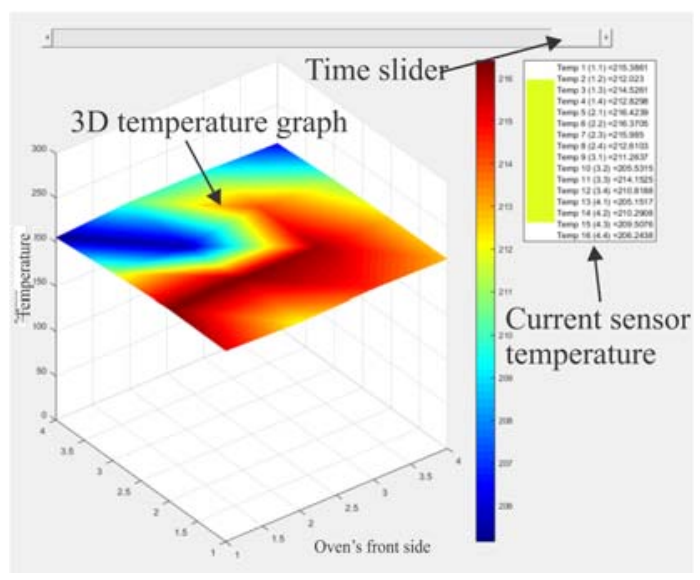

Fig. 83D graph of temperature field inside oven chamber.

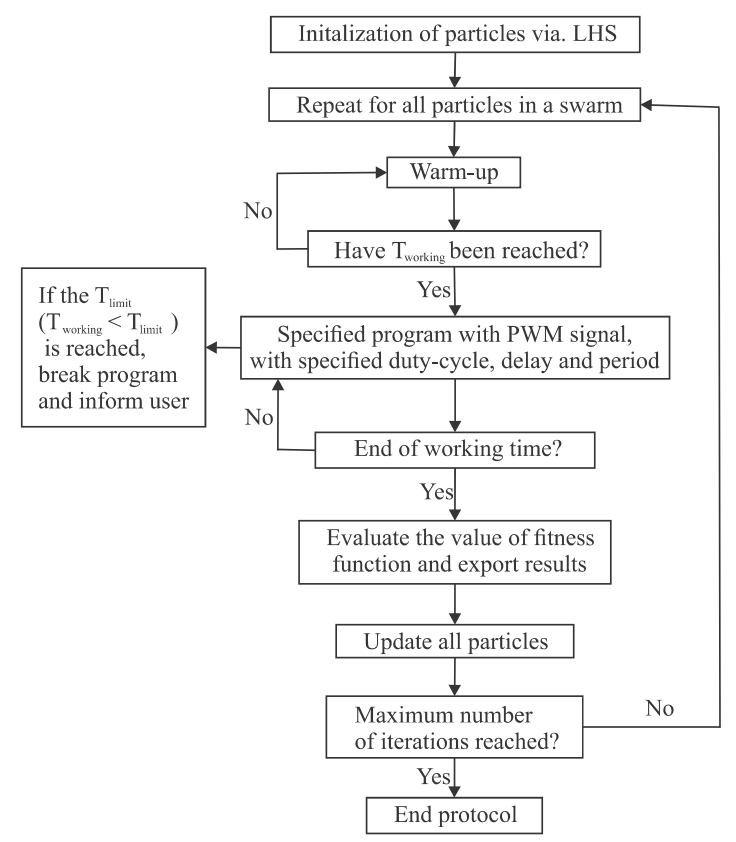

Fig. 9: Workflow of the proposed protocol.

Discussed fitness function (Eq. (4)) is designed in such manner that the PSO algorithm performs the search for ideal baking program, which satisfies the minimal temperature variations around target temperature $200{ }^{\circ} \mathrm{C}$. If for instance no target temperature is choosen, the PSO algorithm would probably find the optimum when the oven 
is turned off (duty-cycle is equal to 0 ) or fully operational (duty-cycle is equal to 1 ).

Visual interface enables user to explore optimization protocol information about temperature field inside oven, PWM signal properties, fitmess function values of desired particle in desired iteration are stated in Fig. 10. and Fig. 11.

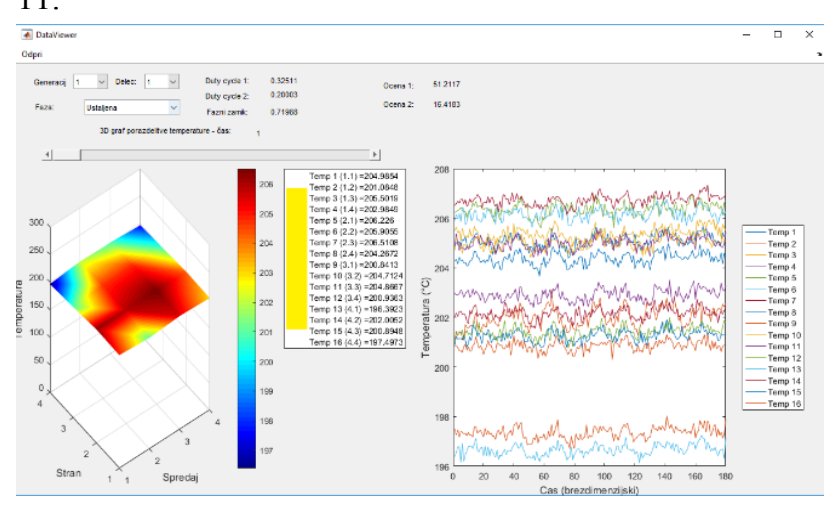

Fig. 10: Visual interface.

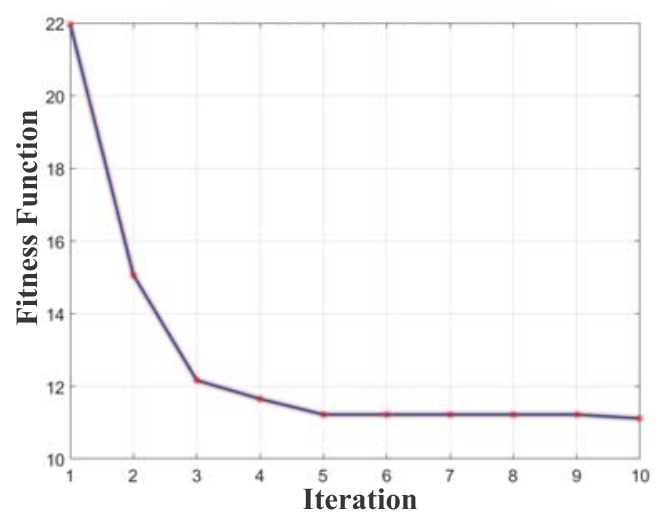

Fig. 11. Convergence of the proposed protocol.

\section{CONCLUSION}

Proposed system is able to accurately depict temperature field inside an oven in real-time. Described protocol is able to manipulate, detect and optimize the temperature field fluctuations inside oven chamber by finding the right sequence of ON/OFF turning the heaters (appropriate PWM signal), in order to achieve uniform temperature distributions. The optimization can potentially be tackled with any heuristic optimization algorithm (ABC, GA, Jaya etc.), where adequate fitness value function is defined. In this study, fitness function used is defined as an absolute difference between target temperature and maximal or minimal temperatures (detected by sensors), when temperatures in the oven chamber settle (start fluctuate around fixed values).

Proposed protocol can be translated to any number of heat sources, to find near optimal PWM signal. Procedure of defining the program with specified PWM signals can be separated into desired number of sub-procedures for more efficient baking operation, offering better products to consumer. Each subprogram needs to be optimized seperatly. To ensure desired results. Fitness function can be replaced with arbitrary function, that represents specific customer requirements.

In the future we plan to include more a fan to manipulate airflow velocity in an oven chamber to increase convective heat transfer and even greater temperature field uniformity.

\section{ACKNOMFDGEMENTS}

This work would not have been possible without support of Faculty of Mechanical Engineering, University of Maribor.

\section{REFERENCES}

[1] Malovany, D. (2011). Proceedings of the 87th Annual Technical Conference, The Journal of American Baking, Chicago.

[2] Popa, B., Popescu, I., M., Popescu, D., Bobaşu, E. (2018). Real-time monitoring system of a closed oven, 19th Internacional Carpahian Control Conference (ICCC) , pp. 27-32.

[3] Kokolj, U., Škerget, L., Ravnik, J. (2017). The validation of numerical methodology for oven design optimization using numerical simulations and baking experiments. Journal of Mechanical Engineering, vol. 63(4), pp. 215-224, DOI: $10.5545 /$ sv-jme. 2016.4089

[4] Marcotte, M. (2007). Heat and mass transfer during baking. WIT transactions on state-of-art in science and engineering, vol. 13, WIT Press, Canada.

[5] Chhanwal, N., Tank, A., Raghavarao, K., Anandharamakrishnan, C. (2012). Computational Fluid Dynamics (CFD) modeling for bread baking proces-A review. Food and Bioprocess Technology, vol. 4, pp. 1157-1172, DOI: 10.1007/s13197-012-0736-6

[6] Pitchai, K., Chen, J., Birla, S., Gonzalez, R., Jones, D., Subbiah, J. (2014). A microvawe heat transfoer model for rotating multi-component meal in domestic oven: Development and validation. Journal of Food Engineering, vol. 10, pp. 60-71, DOI: 10.1016/j.jfoodeng.2013.12.015

[7] Zhang, H., Datta, A.K. (2000). Coupled electromagnetic and thermal modeling of microwave oven heating of foods. Journal of Microwave Power and Elkectromagnetic Energy, vol. 35 (2), pp. 71-85.

[8] Kennedy, J., Eberhart, R. (1995). Particle swarm optimization, Proceedings of the IEEE International Conference on Neural Networks, vol. 4, pp. 1942-1948, DOI: 10.1109/ICNN.1995.488968

[9] Ficko, M.; Brezovnik, S.; Klancnik, S.; Balic, J.; Brezocnik, M.; Pahole, I. (2010). Intelligent Design of an Unconstrained Layout for a Flexible Manufacturing System. Neurocomputing, vol. 73, 639-647, DOI: 10.1016/j.neucom.2009.06.019

[10] Hrelja, M., Klancnik, S., Irgolic, T., Paulic, M., Balic, J., Brezocnik, M. (2014). Turning Parameters 
Optimization using Particle Swarm Optimization. 24Th Daaam International Symposium on Intelligent Manufacturing and Automation, vol. 69, pp. 670-677, DOI: 10.1016/j.proeng.2014.03.041

[11] McKay, M.D., Beckman, R.J., Conover, W.J. (1979). A comparison of three methods for selecting values of input variables in the analysis of output from the computer code. Technometrics, Vol. 21 (2), pp. 239-245, DOI: 10.2307/1268522
[12] Eglajs, V., Audze, P. (1977). New approach to the design of multifactor experiments. Problems of Dynamics and Strengths, vol. 35, pp. 104-107, 1977.

[13] Clerc, M., Kennedy, J. (2002). The Particle Swarm: Explosion, stability and convergence in a multidimensional complex space. IEEE Transactions on Evolutionary Computation, vol. 6, pp. 58-73, DOI: $10.1109 / 4235.985692$. 